\section{Investigations for tuberculosis contacts: what are the real issues of all the efforts?}

\author{
Jean-Pierre Zellweger
}

In this issue of the journal, Cavany et al ${ }^{1}$ present the results of an analysis of contact tracing for TB conducted in London between 2012 and 2015, using cohort review data from the London TB Register, and try to identify some characteristics associated with indicators and yield. The study concludes that contacts of cases with smear-positive pulmonary $\mathrm{TB}$ are identified with active disease in a much higher proportion than contacts of non-pulmonary cases $(4.1 \%$ vs $0.7 \%)$, that the identification of contacts is less frequent among men and former prisoners than among women and persons who were never imprisoned and that the identification of contacts is more likely if the contacts are examined at the same clinic as the index case. Furthermore, $10 \%$ of evaluated children contacts have been identified with latent TB infection.

TB is a transmissible disease, with a slow and unpredictable evolution, from transient or stable latent infection (in the majority of contacts) to active disease in a minority. ${ }^{2}$ Search for secondary cases (persons with another active but still undetected disease among the contacts of an index case) has long been the main reason for investigating the contacts of a TB case, and is still so in many highincidence countries ${ }^{3}$ where resources do not allow more than detection and treatment of active TB cases. Recently, it has been realised that the search for infected contacts, particularly if they belong to a group with an increased risk of progression to $\mathrm{TB}$ who may benefit from a preventive treatment (children, immunocompromised hosts, recently infected adults) is equally warranted and may contribute to the decrease of the pool of future cases. ${ }^{4}$ This policy has been integrated within the new vision of TB elimination as envisaged for low-incidence countries. ${ }^{5}$

Correspondence to Dr Jean-Pierre Zellweger, TB Competence Center, Swiss Lung Association Chutzenstrasse 10, Berne 3000, Switzerland; zellwegerjp@hispeed.ch
In theory, contact tracing should follow simple rules and should detect most persons at risk. International recommendations set indicators for the successful performance of contact tracing. ${ }^{6}$ In practice however, the performance is influenced by many factors, some of them unpredictable and associated with the local traditions, organisation and available human resources, and ease and quality of contacts of the health workers with the index case and the contacts. Some contacts may escape the screening, because of reluctance to be examined by a delegate from a governmental office, by fear of rejection if found infected or because the mere idea of contagion is unknown to them. In some settings, migrants may be less examined than contacts from the local population ${ }^{7}$ probably in part because they are a mobile population with frequent residency change.

The report by Cavany et al demonstrates that it is possible to reach the target of a high proportion of contacts screened and that few factors decrease the yield of contact tracing. It is particularly reassuring to note that migrant status is not an obstacle to contact tracing. On the other side, is also confirms that experienced staff with sufficient resources and time available perform better than overburdened teams. In some places, absence of staff or lack of resources may be the main reason for not performing serious contact investigations, thus missing opportunities to detect new cases (ref). The lesson is that, if contact investigation is recommended in national guidelines, sufficient resources should be made available, particularly if the screening is mainly targeted to the detection of secondary cases and latent infection in children.

Finally, contact investigation should be followed by an appropriate offer of preventive treatment to all infected contacts who may benefit from it, which is not always the case. ${ }^{8}$ The reluctance of medical doctors to prescribe a preventive treatment to persons who are asymptomatic may also play an important role. The study by Cavany et al does not consider this final aspect of the screening procedure, but this should be obvious for all teams performing screening. The prescription of preventive treatment to infected contacts at risk of developing TB (mainly children, immunocompromised persons and recent contacts) is beneficial ${ }^{9}$ even if the current diagnostic tools do not have a sufficient positive predictive value for targeting only those contacts who are at highest risk. New development in this area may decrease the number needed to treat to prevent one case of $\mathrm{TB}$ and avoid useless and potentially harmful treatment. ${ }^{10}$

\section{Competing interests None declared.}

Provenance and peer review Commissioned: internally peer reviewed.

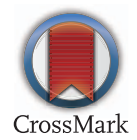

To cite Zellweger J-P. Thorax 2017;72:679.

Published Online First 17 April 2017

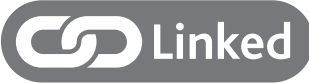

- http://dx.doi.org/10.1136/thoraxjnl-2016-209677

Thorax 2017;72:679. doi:10.1136/

thoraxjnl-2017-210110

\section{REFERENCES}

1 Cavany SM, Sumner T, Vynnycky E et al. An evaluation of tuberculosis contact investigations against national standards. Thorax 2017;72:736-45.

2 Esmail $H$, Barry CE, III, Young DB, et al. The ongoing challenge of latent tuberculosis. Philos Trans $R$ Soc Lond, B, Biol Sci 2014;369:20130437.

3 World Health Organization. Recommendations for investigating contacts of persons with infectious tuberculosis in low- and middle-income countries. Geneva, 2012. Report No.: WHO/HTM/TB/2012.9.

4 World Health Organization. Guidelines on the management of latent tuberculosis infection, WHO 2015. Report No.: WHO/HTM/TB/2015.01.

5 Lönnroth K, Migliori GB, Abubakar l, et al. Towards tuberculosis elimination: an action framework for low-incidence countries. Eur Respir J 2015;45:928-52.

6 Erkens CG, Kamphorst M, Abubakar I, et al. Tuberculosis contact investigation in low prevalence countries: a European consensus. Eur Respir J 2010:36:925-49.

7 Mulder C, van Deutekom H, Huisman EM, et al. Coverage and yield of tuberculosis contact investigations in the Netherlands. Int J Tuberc Lung Dis 2011;15:1630-7.

8 Dobler CC, Luu Q, Marks GB. What patient factors predict physicians' decision not to treat latent tuberculosis infection in tuberculosis contacts? PLOS ONE 2013;8:e76552.

9 Dobler CC, Martin A, Marks GB. Benefit of treatmen of latent tuberculosis infection in individual patients. Eur Respir J 2016:47:1594-5.

10 Petruccioli E, Scriba TJ, Petrone L, et al. Correlates of tuberculosis risk: predictive biomarkers for progression to active tuberculosis. Eur Respir J 2016:48:1751-63. 